\title{
MULTIVALUED ANISOTROPIC PROBLEM WITH FOURIER BOUNDARY CONDITION INVOLVING DIFFUSE RADON MEASURE DATA AND VARIABLE EXPONENTS
}

\author{
Ibrahime Konaté and Stanislas Ouaro \\ Université Joseph Ki Zerbo, Burkina Faso
}

\begin{abstract}
We study a nonlinear anisotropic elliptic problem under Fourier type boundary condition governed by a general anisotropic operator with variable exponents and diffuse Radon measure data which does not charge the sets of zero $p(\cdot)$-capacity. We prove an existence and uniqueness result of entropy or renormalized solution.
\end{abstract}

\section{INTRODUCTION}

Let $\Omega$ be an open bounded domain of $\mathbb{R}^{N}(N \geq 3)$ with smooth boundary $\partial \Omega$ such that meas $(\Omega)>0$. The study of various mathematical problems with variable exponent has received considerable attention in recent years. These problems are very interesting from the purely mathematical point of view. On the other hand, their study is motivated by various applications where such equations appear in the most natural way. These problems arise in many applications as the modeling of electro-rheological fluids which are characterized by their ability to change the mechanical properties under the influence of the exterior electro-magnetic field ([12, 14, 28, 29, 30]), reaction-diffusion systems, modeling of propagation of epidemic disease ([2]). Another important application is the image processing where the anisotropy and nonlinearity of the diffusion operator and convection terms are used to underline the borders of the distorted image and to eliminate the noise $([1,10])$. In this paper, we are

2010 Mathematics Subject Classification. 35J60, 35J65, 35J20, 35J25.

Key words and phrases. Fourier boundary, generalized Lebesgue-Sobolev spaces, anisotropic Sobolev spaces, weak solution, entropy solution, maximal monotone graph, bounded Radon diffuse measure, Marcinkiewicz spaces. 
interested in the following nonlinear multivalued elliptic anisotropic problem:

$$
\left\{\begin{array}{l}
-\sum_{i=1}^{N} \frac{\partial}{\partial x_{i}} a_{i}\left(x, \frac{\partial u}{\partial x_{i}}\right)+\beta(u) \quad \ni \mu \quad \text { in } \Omega, \\
\sum_{i=1}^{N} a_{i}\left(x, \frac{\partial u}{\partial x_{i}}\right) \cdot \eta_{i}+\lambda u=g \quad \text { on } \partial \Omega,
\end{array}\right.
$$

where $\beta$ is a maximal monotone graph on $\mathbb{R}$ such that $0 \in \beta(0), \mu$ a bounded Radon diffuse measure, $|\mu|(\Omega)$ (the total variation of $\mu$ ) a bounded positive measure on $\Omega, g \in L^{1}(\partial \Omega), \lambda>0, \vec{\eta}=\left(\eta_{1}, \ldots, \eta_{N}\right)$ the outward unit normal to $\partial \Omega$.

Note that the space in which we work is the anisotropic Sobolev space $W^{1, \vec{p}(\cdot)}(\Omega)$, where $\vec{p}(\cdot)=\left(p_{1}(\cdot), \ldots, p_{N}(\cdot)\right)$ is a vector with variable components (for $i=1, \ldots, N, p_{i}(\cdot)$ is a continuous function defined below).

We set $\overline{\operatorname{dom}(\beta)}=[m, M]$ with $m \leq 0 \leq M$ and denote by

$$
p_{M}(x):=\max \left(p_{1}(x), \ldots, p_{N}(x)\right) \text { and } p_{m}(x):=\min \left(p_{1}(x), \ldots, p_{N}(x)\right) .
$$

In the classical Lebesgue and Sobolev spaces with constant exponent, many authors have studied problems with a maximal monotone graph and measure data (see $[3,4,5,11,13,19])$. These problems have been extended to the Sobolev spaces with variable exponent in the context of isotropic operators (see $[25,27])$. In this paper, we extend the study of problems with maximal monotone graph and measure data to the Sobolev spaces with variable exponents in the context of anisotropic operators. It is not a surprise to meet new difficulties when passing from isotropic variable exponents to anisotropic variable exponent. The most difficult is the appropriate choice of components in order to obtain necessary a priori estimates. To overcome these difficulties, we combine the classical techniques with the recent techniques that have appeared when treating anisotropic problems with variable exponents.

This paper is focused on the anisotropic elliptic strongly nonlinear equation with variable exponent in which the $\vec{p}(\cdot)$-Laplacian is general. All previous works treating problems like (1.1) considered particular cases of the maximal monotone graph $\beta$ and data $\mu$. Indeed, in [6], Koné et al. used the minimization technique to prove the existence of weak solution when $\beta$ is a power $\left(\beta(t)=|t|^{p_{M}(x)-2} t\right)$ and $\mu$ is an $L^{1}$ function. In [18], Ibrango and Ouaro used the technique of monotone operators in Banach spaces to obtain the existence and uniqueness of entropy solution of problem (1.1) when $\beta$ is a continuous, surjective and nondecreasing function such that $\beta(0)=0$ and $\mu \in L^{1}(\Omega)$.

Our aim is to extend the main result of authors in [18]. More precisely we prove the existence and uniqueness of renormalized or entropy solution to the general elliptic problem (1.1). The novelty in our work is that we are dealing with general non-linearities $\beta$ and measure data. 
We denote by $\mathcal{L}^{N}$ the $N$-dimensional Lebesgue measure of $\mathbb{R}^{N}$ and by $\mathcal{M}_{b}(X)$ the space of bounded Radon measures in $X$, equipped with its standard norm $\|\cdot\|_{\mathcal{M}_{b}(X)}$. Given $\mu \in \mathcal{M}_{b}(X)$, we say that $\mu$ is diffuse with respect to the capacity $W^{1, p(\cdot)}(X)(p(\cdot)$-capacity for short) if $\mu(A)=0$, for every set $A$ such that $\operatorname{Cap}_{p(\cdot)}(A, X)=0$, where the Sobolev $p(\cdot)$-capacity of $A$ with respect to $X$ is defined by

$$
\operatorname{Cap}_{p(\cdot)}(A, X)=\inf _{u \in S_{p(\cdot)}(A)} \int_{X}\left(|u|^{p(x)}+|\nabla u|^{p(x)}\right) d x,
$$

with

$$
\begin{gathered}
S_{p(\cdot)}(A)=\left\{u \in W_{0}^{1, p(\cdot)}(X): u \geq 1 \text { in an open set containing } A\right. \\
\text { and } u \geq 0 \text { in } X\} .
\end{gathered}
$$

In the case $S_{p(\cdot)}(A)=\emptyset$, we set $\operatorname{Cap}_{p(\cdot)}(A, X)=+\infty$.

The set of bounded Radon diffuse measure in the variable exponent setting is denoted by $\mathcal{M}_{b}^{p(\cdot)}(X)$.

Note that, since we are dealing with the Fourier boundary condition, we cannot work with the common space $W_{0}^{1, \vec{p}(\cdot)}(\Omega)$. However, the common space is $W^{1, \vec{p}(\cdot)}(\Omega)$, so we cannot use directly the argument of decomposition of measure, since the second part of the measure is in $W^{-1, p_{m}^{\prime}(\cdot)}(\Omega)$ (the dual of $\left.W_{0}^{1, p_{m}(\cdot)}(\Omega)\right)$.

To overcome this difficulty, we use the same ideas as authors in [27]. We consider a smooth domain $\Omega$ in order to work with the space $W_{0}^{1, \tilde{p}_{m}(\cdot)}\left(U_{\Omega}\right)$, where $\tilde{p}_{m}(\cdot): U_{\Omega} \longrightarrow(1, \infty)$ is a continuous function such that $\tilde{p}_{m}(x)=$ $p_{m}(x)$ for all $x \in \bar{\Omega}$, and return after to the space $W^{1, p_{m}(\cdot)}(\Omega)$. More precisely, $\Omega$ is assumed to be a bounded domain in $\mathbb{R}^{N}$ with a boundary $\partial \Omega$ of class $C^{1}$. Then, $\Omega$ is an extension domain (see [8]), so we can fix an open bounded subset $U_{\Omega}$ of $\mathbb{R}^{N}$ such that $\bar{\Omega} \subset U_{\Omega}$, and there exists a bounded linear operator

$$
E: W^{1, p_{m}(\cdot)}(\Omega) \rightarrow W_{0}^{1, \tilde{p}_{m}(\cdot)}\left(U_{\Omega}\right),
$$

for which

i) $E(u)=u$ a.e. in $\Omega$ for each $u \in W^{1, p_{m}(\cdot)}(\Omega)$,

ii) $\|E(u)\|_{W_{0}^{1, \tilde{p}_{m}(\cdot)}\left(U_{\Omega}\right)} \leq C\|u\|_{W^{1, p_{m}(\cdot)}(\Omega)}$, where $C$ is a constant depending only on $\Omega$.

We define

$$
\mathfrak{M}_{b}^{p_{m}(\cdot)}(\Omega):=\left\{\mu \in \mathcal{M}_{b}^{\tilde{p}_{m}(\cdot)}\left(U_{\Omega}\right): \mu \text { is concentrated on } \Omega\right\} .
$$

This definition is independent of the open set $U_{\Omega}$. Note that for $u \in$ $W^{1, p_{m}(\cdot)}(\Omega) \cap L^{\infty}(\Omega)$ and $\mu \in \mathfrak{M}_{b}^{p_{m}(\cdot)}(\Omega)$, we have

$$
\langle\mu, E(u)\rangle=\int_{\Omega} u d \mu .
$$


On the other hand, as $\mu$ is diffuse, there exist (see [25, 27]) $f \in L^{1}\left(U_{\Omega}\right)$ and $F \in L^{\tilde{p}_{m}^{\prime}(\cdot)}\left(U_{\Omega}\right)^{N}$ such that $\mu=f-\operatorname{div}(F)$ in $\mathcal{D}^{\prime}\left(U_{\Omega}\right)$. Therefore, we can also write

$$
\langle\mu, E(u)\rangle=\int_{U_{\Omega}} f E(u) d x+\int_{U_{\Omega}} F \cdot \nabla E(u) d x .
$$

Before presenting our main result, we first give the following hypotheses.

Let $\vec{p}(\cdot)=\left(p_{1}(\cdot), \ldots, p_{N}(\cdot)\right)$ be such that for any $i=1, \ldots, N, p_{i}(\cdot)$ : $\bar{\Omega} \longrightarrow \mathbb{R}$ is a continuous function with

$$
1<p_{i}^{-}:=\inf _{x \in \bar{\Omega}} p_{i}(x) \leq p_{i}^{+}:=\sup _{x \in \bar{\Omega}} p_{i}(x)<\infty .
$$

The operator $a_{i}: \Omega \times \mathbb{R} \rightarrow \mathbb{R}$ is a Carathéodory function (i.e. $a_{i}(x, \xi)$ is continuous in $\xi$ for a.e. $x \in \Omega$ and measurable in $x$ for every $\xi \in \mathbb{R}$ ) satisfying:

- there exists a positive constant $C_{1}$ such that

$$
\left|a_{i}(x, \xi)\right| \leq C_{1}\left(j_{i}(x)+|\xi|^{p_{i}(x)-1}\right),
$$

for almost every $x \in \Omega$ and for every $\xi \in \mathbb{R}$, where $j_{i}$ is a non-negative function in $L^{p_{i}^{\prime}(\cdot)}(\Omega)$, with $\frac{1}{p_{i}(x)}+\frac{1}{p_{i}^{\prime}(x)}=1$;

- for $\xi, \eta \in \mathbb{R}$ with $\xi \neq \eta$ and for every $x \in \Omega$, there exists a positive constant $C_{2}$ such that

$$
\left(a_{i}(x, \xi)-a_{i}(x, \eta)\right)(\xi-\eta) \geq \begin{cases}C_{2}|\xi-\eta|^{p_{i}(x)} & \text { if }|\xi-\eta| \geq 1 \\ C_{2}|\xi-\eta|^{p_{i}^{-}} & \text {if }|\xi-\eta|<1\end{cases}
$$

- there exists a positive constant $C_{3}$ such that

$$
a_{i}(x, \xi) \cdot \xi \geq C_{3}|\xi|^{p_{i}(x)},
$$

for $\xi \in \mathbb{R}$ and almost every $x \in \Omega$.

The hypotheses on $a_{i}$ are classical in the study of nonlinear PDEs (see $[6,7$, 17]).

Throughout this paper, we assume that

$$
\frac{\bar{p}(N-1)}{N(\bar{p}-1)}<p_{i}^{-}<\frac{\bar{p}(N-1)}{N-\bar{p}}, \frac{p_{i}^{+}-p_{i}^{-}-1}{p_{i}^{-}}<\frac{\bar{p}-N}{\bar{p}(N-1)}
$$

and

$$
\sum_{i=1}^{N} \frac{1}{p_{i}^{-}}>1
$$

where $\frac{N}{\bar{p}}=\sum_{i=1}^{N} \frac{1}{p_{i}^{-}}$, and for all $x \in \partial \Omega$,

$$
p^{\partial}(x)= \begin{cases}\frac{(N-1) p(x)}{N-p(x)} & \text { if } p(x)<N \\ +\infty & \text { if } p(x) \geq N\end{cases}
$$


A prototype example that is covered by our assumption is the following anisotropic $\vec{p}(\cdot)$-Laplacian problem: setting

$$
a_{i}(x, \xi)=|\xi|^{p_{i}(x)-2} \xi \text {, where } p_{i}(x) \geq 2 \text { for any } i=1, \ldots, N,
$$

we obtain the problem

$$
\begin{cases}\beta(u)-\sum_{i=1}^{N} \frac{\partial}{\partial x_{i}}\left(\left|\frac{\partial u}{\partial x_{i}}\right|^{p_{i}(x)-2} \frac{\partial u}{\partial x_{i}}\right) \ni \mu & \text { in } \Omega, \\ \sum_{i=1}^{N} a_{i}\left(x, \frac{\partial u}{\partial x_{i}}\right) \cdot \eta_{i}+\lambda u=g & \text { on } \partial \Omega .\end{cases}
$$

For any $l_{0}>0$, we consider a function $h_{0}$ such that

(i) $h_{0} \in C_{c}^{1}(\mathbb{R}), h_{0}(r) \geq 0$, for all $r \in \mathbb{R}$,

(ii) $h_{0}(r)=1$ if $|r| \leq l_{0}$ and $h_{0}(r)=0$ if $|r| \geq l_{0}+1$.

If $\gamma$ is a maximal monotone operator defined on $\mathbb{R}$, we denote by $\gamma_{0}$ the main section of $\gamma$; i.e.,

$$
\gamma_{0}(s)= \begin{cases}\text { minimal absolute value of } \gamma(s) & \text { if } \gamma(s) \neq \emptyset, \\ +\infty & \text { if }[s,+\infty) \cap \operatorname{dom}(\gamma)=\emptyset, \\ -\infty & \text { if }(-\infty, s] \cap \operatorname{dom}(\gamma)=\emptyset .\end{cases}
$$

We give a useful convergence result (see [25]).

LEMMA 1.1. Let $\left(\beta_{n}\right)_{n>1}$ be a sequence of maximal monotone graphs such that $\beta_{n} \rightarrow \beta$ in the sense of the graph (for $(x, y) \in \beta$, there exists $\left(x_{n}, y_{n}\right) \in \beta_{n}$ such that $x_{n} \rightarrow x$ and $\left.y_{n} \rightarrow y\right)$. We consider two sequences $\left(z_{n}\right)_{n \geq 1} \subset L^{1}(\Omega)$ and $\left(w_{n}\right)_{n \geq 1} \subset L^{1}(\Omega)$. We suppose that: $\forall n \geq 1, w_{n} \in \beta_{n}\left(z_{n}\right),\left(w_{n}\right)_{n \geq 1}$ is bounded in $L^{1}(\Omega)$ and $z_{n} \rightarrow z$ in $L^{1}(\Omega)$. Then,

$$
z \in \operatorname{dom}(\beta) \text {. }
$$

The rest of the paper is organized as follows. In Section 2, we introduce some fundamental preliminary results which are useful in this work. Then, we study the existence and uniqueness of entropy or renormalized solution in Section 3.

\section{Preliminary Results}

We recall in this section some definitions and basic properties of anisotropic Lebesgue and Sobolev spaces with variable exponents. Set

$$
C_{+}(\bar{\Omega})=\left\{p \in C(\bar{\Omega}): \min _{x \in \bar{\Omega}} p(x)>1\right\} .
$$

For any $p \in C_{+}(\bar{\Omega})$, the variable exponent Lebesgue space is defined by $L^{p(\cdot)}(\Omega):=\left\{u: \Omega \rightarrow \mathbb{R}\right.$ a measurable function such that $\left.\int_{\Omega}|u|^{p(x)} d x<\infty\right\}$, 
endowed with the so-called Luxemburg norm

$$
|u|_{p(\cdot)}:=\inf \left\{\lambda>0: \int_{\Omega}\left|\frac{u(x)}{\lambda}\right|^{p(x)} d x \leq 1\right\} .
$$

The $p(\cdot)$-modular of the space $L^{p(\cdot)}(\Omega)$ is the mapping $\rho_{p(\cdot)}: L^{p(\cdot)}(\Omega) \longrightarrow \mathbb{R}$ defined by

$$
\rho_{p(\cdot)}(u):=\int_{\Omega}|u|^{p(x)} d x .
$$

For any $u \in L^{p(\cdot)}(\Omega)$, the following inequality (see $[15,16]$ ) will be used later:

$$
\min \left\{|u|_{p(\cdot)}^{p^{-}} ;|u|_{p(\cdot)}^{p^{+}}\right\} \leq \rho_{p(\cdot)}(u) \leq \max \left\{|u|_{p(\cdot)}^{p^{-}} ;|u|_{p(\cdot)}^{p^{+}}\right\} .
$$

For any $u \in L^{p(\cdot)}(\Omega)$ and $v \in L^{q(\cdot)}(\Omega)$, with $\frac{1}{p(x)}+\frac{1}{q(x)}=1$ for any $x \in \Omega$, we have the Hölder type inequality

$$
\left|\int_{\Omega} u v d x\right| \leq\left(\frac{1}{p^{-}}+\frac{1}{q^{-}}\right)|u|_{p(\cdot)}|v|_{q(\cdot)} .
$$

If $\Omega$ is bounded and $p, q \in C_{+}(\bar{\Omega})$ such that $p(x) \leq q(x)$ for any $x \in \Omega$, then the embedding $L^{p(\cdot)}(\Omega) \hookrightarrow L^{q(\cdot)}(\Omega)$ is continuous (see [23, Theorem 2.8]).

Herein, we need the following anisotropic Sobolev space with variable exponent:

$$
W^{1, \vec{p}(\cdot)}(\Omega):=\left\{u \in L^{p_{M}(\cdot)}(\Omega): \frac{\partial u}{\partial x_{i}} \in L^{p_{i}(\cdot)}(\Omega), i=1, \ldots, N\right\},
$$

which is a separable and reflexive Banach space (see [24]) under the norm

$$
\|u\|_{\vec{p}(\cdot)}=|u|_{p_{M}(\cdot)}+\sum_{i=1}^{N}\left|\frac{\partial u}{\partial x_{i}}\right|_{p_{i}(\cdot)} .
$$

We need the following embedding and trace results.

TheOREM 2.1 ([15, Corollary 2.1]). Let $\Omega \subset \mathbb{R}^{N}(N \geq 3)$ be a bounded open set and for all $i=1, \ldots, N, p_{i} \in L^{\infty}(\Omega), p_{i}(x) \geq 1$ a.e. $x \in \Omega$. Then, for any $q \in L^{\infty}(\Omega)$ with $q(x) \geq 1$ a.e. $x \in \Omega$ such that

$$
\text { ess } \inf _{x \in \Omega}\left(p_{M}(x)-q(x)\right)>0,
$$

we have the compact embedding

$$
W^{1, \vec{p}(\cdot)}(\Omega) \hookrightarrow L^{q(\cdot)}(\Omega) .
$$

TheOREM 2.2 ([7, Theorem 6$])$. Let $\Omega \subset \mathbb{R}^{N}(N \geq 3)$ be a bounded open set with smooth boundary and let $\vec{p}(\cdot) \in\left(C_{+}(\bar{\Omega})\right)^{N}, r \in C(\bar{\Omega})$ satisfy the condition

$$
1 \leq r(x)<\min \left\{p_{1}^{\partial}(x), \ldots, p_{N}^{\partial}(x)\right\}, \quad \forall x \in \partial \Omega
$$


Then, there exists a compact boundary trace embedding

$$
W^{1, \vec{p}(\cdot)}(\Omega) \hookrightarrow L^{r(\cdot)}(\partial \Omega) .
$$

In particular,

$$
W^{1, \vec{p}(\cdot)}(\Omega) \hookrightarrow L^{1}(\partial \Omega) .
$$

We introduce now the numbers

$$
q=\frac{N(\bar{p}-1)}{N-1} \text { and } q^{*}=\frac{N(\bar{p}-1)}{N-\bar{p}}=\frac{N q}{N-q} .
$$

The following result is due to Troisi (see [31]).

Theorem 2.3. Let $p_{1}, \ldots, p_{N} \in[1, \infty) ; g \in W^{1,\left(p_{1}, \ldots, p_{N}\right)}(\Omega)$ and

$$
\left\{\begin{array}{l}
q=(\bar{p})^{*} \quad \text { if }(\bar{p})^{*}<N \\
q \in[1, \infty) \quad \text { if }(\bar{p})^{*} \geq N .
\end{array}\right.
$$

Then, there exists a constant $C_{4}>0$ depending on $N, p_{1}, \ldots, p_{N}$ if $\bar{p}<N$ and also on $q$ and $\operatorname{meas}(\Omega)$ if $\bar{p} \geq N$ such that

$$
\|g\|_{L^{q}(\Omega)} \leq C_{4} \prod_{i=1}^{N}\left[\|g\|_{L^{p_{M}(\Omega)}}+\left\|\frac{\partial g}{\partial x_{i}}\right\|_{L^{p_{i}(\Omega)}}\right]^{\frac{1}{N}},
$$

where $\frac{1}{\bar{p}}=\sum_{i=1}^{N} p_{i}$ and $(\bar{p})^{*}=\frac{N \bar{p}}{N-\bar{p}}$.

In this paper, we will use the Marcinkiewicz space $\mathcal{M}^{q}(\Omega)(1<q<+\infty)$ as the set of measurable function $g: \Omega \longrightarrow \mathbb{R}$ for which the distribution

$$
\lambda_{g}(k):=\operatorname{meas}(\{x \in \Omega:|g(x)|>k\}), k \geq 0
$$

satisfies an estimate of the form

$$
\lambda_{g}(k) \leq C k^{-q}, \text { for some finite constant } C>0 .
$$

We will use the following pseudo norm in $\mathcal{M}^{q}(\Omega)$.

$$
\|g\|_{\mathcal{M}^{q}(\Omega)}:=\inf \left\{C>0: \lambda_{g}(k) \leq C k^{-q}, \forall k>0\right\} .
$$

Finally, we will use through the paper, the truncation function $T_{k}(k>0)$, defined by

$$
T_{k}(s)=\max \{-k, \min \{k ; s\}\} .
$$

It is clear that $\lim _{k \rightarrow+\infty} T_{k}(s)=s$ and $\left|T_{k}(s)\right|=\min \{|s| ; k\}$.

For any $v \in W^{1, \vec{p}(\cdot)}(\Omega)$, we use $v$ instead of $\left.v\right|_{\partial \Omega}$ for the trace of $v$ on $\partial \Omega$. Set $\mathcal{T}^{1, \vec{p}(\cdot)}(\Omega)$ as the set of the measurable functions $u: \Omega \longrightarrow \mathbb{R}$ such that for any $k>0, T_{k}(u) \in W^{1, \vec{p}(\cdot)}(\Omega)$. We define the space $\mathcal{T}_{t r}^{1, \vec{p}(\cdot)}(\Omega)$ as the set of functions $u \in \mathcal{T}^{1, \vec{p}(\cdot)}(\Omega)$ such that there exists a sequence $\left(u_{n}\right)_{n \in \mathbb{N}} \subset$ $W^{1, \vec{p}(\cdot)}(\Omega)$ satisfying: 
i) $u_{n} \longrightarrow u$ a.e. in $\Omega$ as $n \rightarrow+\infty$,

ii) $\frac{\partial T_{k}\left(u_{n}\right)}{\partial x_{i}} \longrightarrow \frac{\partial T_{k}(u)}{\partial x_{i}}$ in $L^{1}(\Omega)$, for all $k>0$ as $n \rightarrow+\infty$,

iii) there exists a measurable function $v$ on $\partial \Omega$ such that $u_{n} \longrightarrow v$ a.e. on $\partial \Omega$ as $n \rightarrow+\infty$.

We need the following lemma proved in [6].

LEMMA 2.4. Let $g$ be a nonnegative function in $W^{1, \vec{p}(\cdot)}(\Omega)$. Assume $\bar{p}<N$ and there exists a constant $C>0$ such that

$$
\int_{\Omega}\left|T_{k}(g)\right|^{p_{M}^{-}} d x+\sum_{i=1}^{N} \int_{\{|g| \leq k\}}\left|\frac{\partial g}{\partial x_{i}}\right|^{p_{i}^{-}} d x \leq C(k+1),
$$

for every $k>0$. Then, there exists a constant $D$, depending on $C$ such that

$$
\|g\|_{\mathcal{M}^{q^{*}}(\Omega)} \leq D
$$

where $q^{*}=\frac{N(\bar{p}-1)}{N-\bar{p}}$

\section{Statement of the main RESUlts}

The notion of renormalized solution to problem (1.1) where the data $\mu$ belongs to $\mathfrak{M}_{b}^{p_{m}(\cdot)}(\Omega)$ is the following.

Definition 3.1. For any $\mu \in \mathfrak{M}_{b}^{p_{m}(\cdot)}(\Omega)$ and $g \in L^{1}(\partial \Omega)$, a renormalized solution of problem (1.1) is a couple $(u, b) \in \mathcal{T}_{t r}^{1, \vec{p}(\cdot)}(\Omega) \times L^{1}(\Omega)$, $u \in \operatorname{dom}(\beta) \mathcal{L}^{N}$ - a.e. in $\Omega, b \in \beta(u) \mathcal{L}^{N}$ - a.e. in $\Omega, \operatorname{tr}(u) \in L^{1}(\partial \Omega)$, there exists $\nu \in \mathcal{M}_{b}^{p_{m}(\cdot)}(\Omega)$ such that $\nu \perp \mathcal{L}^{N}$,

$$
\nu^{+} \text {is concentred on }[u=M], \nu^{-} \text {is concentred on }[u=m]
$$

and

$$
\begin{aligned}
& \sum_{i=1}^{N} \int_{\Omega} a_{i}\left(x, \frac{\partial u}{\partial x_{i}}\right) \frac{\partial \varphi}{\partial x_{i}} d x+\int_{\Omega} b \varphi d x+\int_{\Omega} \varphi d \nu+\lambda \int_{\partial \Omega} u \varphi d \sigma \\
& \quad=\int_{\Omega} \varphi d \mu+\int_{\partial \Omega} g \varphi d \sigma,
\end{aligned}
$$

for any $\varphi \in W^{1, \vec{p}(\cdot)}(\Omega) \cap L^{\infty}(\Omega)$. Moreover,

$$
\lim _{n \rightarrow+\infty} \int_{[n \leq|u| \leq n+1]}\left|\frac{\partial u}{\partial x_{i}}\right|^{p_{i}(x)} d x=0, \text { for } i=1, \ldots, N .
$$

Theorem 3.2. Assume that (1.2)-(1.7) hold, $\mu \in \mathfrak{M}_{b}^{p_{m}(\cdot)}(\Omega)$ and $g \in$ $L^{1}(\partial \Omega)$. Then, problem (1.1) admits a renormalized solution. 
Proof. The proof is done in three steps.

STEP 1 (the approximate problem). For every $\epsilon>0$, we consider the Yosida regularization $\beta_{\epsilon}$ of $\beta$ (see [9]), given by

$$
\beta_{\epsilon}=\frac{1}{\epsilon}\left(I-(I+\epsilon \beta)^{-1}\right) \text {. }
$$

Thanks to [9], there exists a non negative, convex and l.s.c. function $j$ defined on $\mathbb{R}$ such that

$$
\beta=\partial j
$$

To regularise $\beta$, we consider

$$
j_{\epsilon}(s)=\min _{r \in \mathbb{R}}\left\{\frac{1}{2 \epsilon}|s-r|^{2}+j(r)\right\}, \forall s \in \mathbb{R}, \forall \epsilon>0 .
$$

By [9, Proposition 2.11] we have

$$
\left\{\begin{array}{l}
\operatorname{dom}(\beta) \subset \operatorname{dom}(j) \subset \overline{\operatorname{dom}(j)}=\overline{\operatorname{dom}(\beta)}, \\
j_{s}(s)=\frac{\epsilon}{2}\left|\beta_{\epsilon}(s)\right|^{2}+j\left(J_{\epsilon}\right) \text { where } J_{\epsilon}:=(I+\epsilon \beta)^{-1}, \\
j_{\epsilon} \text { is a convex, Frechet-differentiable function and } \beta_{\epsilon}=\partial j_{\epsilon}, \\
j_{\epsilon} \uparrow j \text { as } \epsilon \downarrow 0 .
\end{array}\right.
$$

Moreover, for any $\epsilon>0, \beta_{\epsilon}$ is a nondecreasing and Lipschitz-continuous function (see [27]).

Since $\mu \in \mathcal{M}_{b}^{\tilde{p}_{m}(\cdot)}\left(U_{\Omega}\right)$, recall that $\mu=f-\operatorname{div}(F)$ in $\mathcal{D}^{\prime}\left(U_{\Omega}\right)$ with $f \in$ $L^{1}\left(U_{\Omega}\right)$ and $F \in\left(L^{\tilde{p}_{m}^{\prime}(\cdot)}\left(U_{\Omega}\right)\right)^{N}$ where $U_{\Omega}$ is the open subset of $\mathbb{R}^{N}$ which extends $\Omega$ via the operator $E$.

We regularize $f, g$ and $\mu$ respectively as follows. For any $\epsilon>0$ and $x \in U_{\Omega}$, we define the functions

$$
f_{\epsilon}(x)=T_{\frac{1}{\epsilon}}(f(x)) \chi_{\Omega}(x), \quad g_{\epsilon}(x)=T_{\frac{1}{\epsilon}}(g(x)) \chi_{\partial \Omega}(x) .
$$

Let $\left(F_{\epsilon}\right)_{\epsilon>1} \subset C_{0}^{\infty}\left(U_{\Omega}\right)$ be a sequence such that $F_{\epsilon} \rightarrow F$ strongly in $\left(L^{\tilde{p}_{m}^{\prime}(\cdot)}\left(U_{\Omega}\right)\right)^{N}$. For any $\epsilon>0$, we set $\tilde{F}_{\epsilon}=\chi_{\Omega} F_{\epsilon}$ and $\mu_{\epsilon}=f_{\epsilon}-\operatorname{div}\left(\tilde{F}_{\epsilon}\right)$. For any $\epsilon>0$, one has

- $\mu_{\epsilon} \in \mathfrak{M}_{b}^{p_{m}(\cdot)}(\Omega), \mu_{\epsilon} \rightarrow \mu$ in $\mathcal{M}_{b}\left(U_{\Omega}\right)$ and $\mu_{\epsilon} \in L^{\infty}(\Omega)$,

- $\left(f_{\epsilon}\right)_{\epsilon>0}$ and $\left(g_{\epsilon}\right)_{\epsilon>0}$ are sequences of bounded functions which converges to $f \in L^{1}(\Omega)$ and $g \in L^{1}(\partial \Omega)$ respectively.

Moreover,

$$
\left\|f_{\epsilon}\right\|_{L^{1}(\Omega)} \leq\|f\|_{L^{1}(\Omega)}, \forall \epsilon>0, \quad\left\|g_{\epsilon}\right\|_{L^{1}(\partial \Omega)} \leq\|g\|_{L^{1}(\partial \Omega)}, \quad \forall \epsilon>0
$$

and

$$
\left|\int_{\Omega} T_{k}(\varphi) d \mu_{\epsilon}\right| \leq k C(\mu, \Omega), \forall k>0, \forall \varphi \in \mathcal{T}^{1, p_{m}(\cdot)}(\Omega) .
$$

We have the following lemma (see [27, Lemma 4.1]).

Lemma 3.3. The Yosida regularization $\beta_{\epsilon}$ is a surjective operator. 
Now, we consider the following approximating scheme problem

$$
P\left(\beta_{\epsilon}, \mu_{\epsilon}\right) \begin{cases}-\sum_{i=1}^{N} \frac{\partial}{\partial x_{i}} a_{i}\left(x, \frac{\partial u_{\epsilon}}{\partial x_{i}}\right)+\beta_{\epsilon}\left(u_{\epsilon}\right)+\epsilon\left|u_{\epsilon}\right|^{P_{M}(x)-2} u_{\epsilon}=\mu_{\epsilon} & \text { in } \Omega \\ \sum_{i=1}^{N} a_{i}\left(x, \frac{\partial u_{\epsilon}}{\partial x_{i}}\right) \cdot \eta_{i}+\lambda u_{\epsilon}=g_{\epsilon} & \text { on } \partial \Omega\end{cases}
$$

where $\epsilon>0$.

THEOREM 3.4. The problem (3.4) admits at least one weak solution $u_{\epsilon}$ in the sense that $u_{\epsilon} \in W^{1, \vec{p}(\cdot)}(\Omega) \cap L^{1}(\partial \Omega), \beta_{\epsilon}\left(u_{\epsilon}\right) \in L^{\infty}(\Omega)$ and $\forall \varphi \in$ $W^{1, \vec{p}(\cdot)}(\Omega) \cap L^{\infty}(\Omega)$,

$$
\begin{aligned}
& \sum_{i=1}^{N} \int_{\Omega} a_{i}\left(x, \frac{\partial u_{\epsilon}}{\partial x_{i}}\right) \frac{\partial \varphi}{\partial x_{i}} d x+\int_{\Omega} \beta_{\epsilon}\left(u_{\epsilon}\right) \varphi d x+\epsilon \int_{\Omega}\left|u_{\epsilon}\right|^{P_{M}(x)-2} u_{\epsilon} \varphi d x \\
& +\lambda \int_{\partial \Omega} u_{\epsilon} \varphi d \sigma=\int_{\Omega} \varphi d \mu_{\epsilon}+\int_{\partial \Omega} g_{\epsilon} \varphi d \sigma .
\end{aligned}
$$

Proof. If $b$ is a surjective, continuous and nondecreasing function with $b(0)=0$ and $\Upsilon \in L^{\infty}(\Omega)$, for any $k>0$, the following problem

$$
P\left(T_{k}(b), \Upsilon\right) \begin{cases}-\sum_{i=1}^{N} \frac{\partial}{\partial x_{i}} a_{i}\left(x, \frac{\partial u}{\partial x_{i}}\right)+T_{k}(b(u))+\epsilon|u|^{P_{M}(x)-2} u=\Upsilon & \text { in } \Omega, \\ \sum_{i=1}^{N} a_{i}\left(x, \frac{\partial u}{\partial x_{i}}\right) \cdot \eta_{i}+\lambda T_{k}(u)=g & \text { on } \partial \Omega\end{cases}
$$

admits at least one solution $u_{k} \in W^{1, \vec{p}(\cdot)}(\Omega)$ such that for all $\varphi \in$ $W^{1, \vec{p}(\cdot)}(\Omega) \cap L^{\infty}(\Omega)$,

$$
\begin{aligned}
& \sum_{i=1}^{N} \int_{\Omega} a_{i}\left(x, \frac{\partial u_{k}}{\partial x_{i}}\right) \frac{\partial \varphi}{\partial x_{i}} d x+\int_{\Omega} T_{k}\left(b\left(u_{k}\right)\right) \varphi d x+\epsilon \int_{\Omega}\left|u_{k}\right|^{P_{M}(x)-2} u_{k} \varphi d x \\
& \quad+\lambda \int_{\partial \Omega} T_{k}\left(u_{k}\right) \varphi d \sigma=\int_{\Omega} \Upsilon \varphi d x+\int_{\partial \Omega} g \varphi d \sigma .
\end{aligned}
$$

Furthermore,

$$
\forall k>\|\Upsilon\|_{\infty}, \quad\left|b\left(u_{\epsilon}\right)\right| \leq\|\Upsilon\|_{\infty} \text { a.e. in } \Omega .
$$

Indeed, we define an operator $A_{k}$ by

$$
\left\langle A_{k}(u), \varphi\right\rangle=\langle A(u), \varphi\rangle+\int_{\partial \Omega} T_{k}(b(u)) \varphi d x+\lambda \int_{\partial \Omega} T_{k}(u) \varphi d \sigma, \forall u, \varphi \in X_{0},
$$


where

$$
\langle A(u), \varphi\rangle=\sum_{i=1}^{N} \int_{\Omega} a_{i}\left(x, \frac{\partial u}{\partial x_{i}}\right) \frac{\partial \varphi}{\partial x_{i}} d x+\epsilon \int_{\Omega}|u|^{p_{M}(x)-2} u \varphi d x .
$$

We also define the reflexive space

$$
E:=W^{1, \vec{p}(\cdot)}(\Omega) \times L^{p_{M}(\cdot)}(\partial \Omega) .
$$

Let $X_{0}$ be the subspace of $E$ defined by

$$
X_{0}=\{(u, v) \in E: v=\tau(u)\},
$$

where $\tau(u)$ is the trace of $u \in \mathcal{T}_{t r}^{1, \vec{p}(\cdot)}(\Omega)$ in the usual sense, since $u \in$ $W^{1, \vec{p}(\cdot)}(\Omega)$. In the sequel, we will identify an element $(u, v) \in X_{0}$ with its representative $u \in W^{1, \vec{p}(\cdot)}(\Omega)$.

The operator $A_{k}$ is onto (see $[17,18]$ ). Therefore, by setting

$$
\langle F, \varphi\rangle=\int_{\Omega} \Upsilon \varphi d x+\int_{\partial \Omega} g \varphi d x
$$

it follows that $F \in E^{\prime} \subset X_{0}^{\prime}$. Then, we can deduce the existence of a function $u_{k} \in X_{0}$ such that

$$
\left\langle A_{k}\left(u_{k}\right), \varphi\right\rangle=\langle F, \varphi\rangle, \text { for all } \varphi \in X_{0} .
$$

We can reason like authors in [26] (see also [18]) to obtain

$$
\left|b\left(u_{k}\right)\right| \leq\|\Upsilon\|_{L^{\infty}(\partial \Omega)}
$$

and

$$
\left|u_{k}\right| \leq \frac{1}{\lambda}\left\|g_{\epsilon}\right\|_{L^{\infty}(\Omega)} .
$$

Since $\left|g_{\epsilon}\right| \leq|g| \Rightarrow\left\|g_{\epsilon}\right\|_{\infty} \leq|g|$, we have

$$
\operatorname{meas}(\partial \Omega) \times\left\|g_{\epsilon}\right\|_{L^{\infty}(\Omega)} \leq\|g\|_{L^{1}(\partial \Omega)} .
$$

Hence, we deduce that

$$
\left\|g_{\epsilon}\right\|_{\left.L^{\infty}(\partial \Omega)\right)} \leq \frac{\|g\|_{L^{1}(\partial \Omega)}}{\operatorname{meas}(\partial \Omega)} .
$$

Now, we fix $k=\max \left(\|\Upsilon\|_{L^{\infty}(\partial \Omega)}, \frac{\|g\|_{L^{1}(\partial \Omega)}}{\lambda \operatorname{meas}(\partial \Omega)}\right)+1$ in $P\left(T_{k}(b), \Upsilon\right)$ and set $\Upsilon=\mu_{\epsilon}, b=\beta_{\epsilon}$ to end the proof of Theorem 3.4.

Lemma 3.5. Let $u_{\epsilon}$ be a weak solution of $P\left(\beta_{\epsilon}, \mu_{\epsilon}\right)$. Then, there exists a positive constant $C(\mu, \Omega)$ such that for any $k>0$,

$$
\begin{gathered}
\sum_{i=1}^{N} \int_{\Omega}\left|\frac{\partial}{\partial x_{i}} T_{k}\left(u_{\epsilon}\right)\right|^{p_{i}(x)} d x \leq k\left(\frac{C(\mu, g, \Omega)+\|g\|_{L^{1}(\partial \Omega)}}{C_{3}}\right), \\
\int_{\Omega} \beta_{\epsilon}\left(u_{\epsilon}\right) T_{k}\left(u_{\epsilon}\right) d x \leq k\left(C(\mu, g, \Omega)+\|g\|_{L^{1}(\partial \Omega)}\right)
\end{gathered}
$$


and

$$
\left\|u_{\epsilon}\right\|_{L^{1}(\partial \Omega)} \leq \frac{C(\mu, \Omega)+\|g\|_{L^{1}(\partial \Omega)}}{\lambda} .
$$

Proof. We begin by proving (3.8) and (3.9). By taking $\varphi=T_{k}\left(u_{\epsilon}\right)$ as test function in (3.5), we get

$$
\begin{gathered}
\sum_{i=1}^{N} \int_{\Omega} a_{i}\left(x, \frac{\partial u_{\epsilon}}{\partial x_{i}}\right) \frac{\partial T_{k}\left(u_{\epsilon}\right)}{\partial x_{i}} d x+\int_{\Omega} \beta_{\epsilon}\left(u_{\epsilon}\right) T_{k}\left(u_{\epsilon}\right) d x \\
+\epsilon \int_{\Omega}\left|u_{\epsilon}\right|^{P_{M}(x)-2} u_{\epsilon} T_{k}\left(u_{\epsilon}\right) d x+\lambda \int_{\partial \Omega} u_{\epsilon} T_{k}\left(u_{\epsilon}\right) d \sigma \\
\quad=\int_{\Omega} T_{k}\left(u_{\epsilon}\right) d \mu_{\epsilon}+\int_{\partial \Omega} g_{\epsilon} T_{k}\left(u_{\epsilon}\right) d \sigma
\end{gathered}
$$

Then, taking into account that $\int_{\partial \Omega} g_{\epsilon} T_{k}\left(u_{\epsilon}\right) d \sigma \leq k\|g\|_{L^{1}(\partial \Omega)}$, we use (1.5) and (3.3) in the last equality to get

$$
\begin{gathered}
C_{3} \sum_{i=1}^{N} \int_{\left|u_{\epsilon}\right| \leq k}\left|\frac{\partial}{\partial x_{i}} T_{k}\left(u_{\epsilon}\right)\right|^{p_{i}(x)} d x+\int_{\Omega} \beta_{\epsilon}\left(u_{\epsilon}\right) T_{k}\left(u_{\epsilon}\right) d x \\
+\lambda \int_{\partial \Omega} u_{\epsilon} T_{k}\left(u_{\epsilon}\right) d \sigma+\epsilon \int_{\Omega}\left|u_{\epsilon}\right|^{P_{M}(x)-2} u_{\epsilon} T_{k}\left(u_{\epsilon}\right) d x \\
\leq k\left(C(\mu, \Omega)+\|g\|_{L^{1}(\partial \Omega)}\right) .
\end{gathered}
$$

Since $T_{k}, \beta_{\epsilon}, s \mapsto|s|^{r(\cdot)-2} s$ are nondecreasing and $\beta_{\epsilon}(0)=T_{k}(0)=0$, all the integrals in (3.11) are nonnegative. Therefore, we deduce from (3.11) that

$$
\sum_{i=1}^{N} \int_{\Omega}\left|\frac{\partial}{\partial x_{i}} T_{k}\left(u_{\epsilon}\right)\right|^{p_{i}(x)} d x \leq k\left(\frac{C(\mu, \Omega)+\|g\|_{L^{1}(\partial \Omega)}}{C_{3}}\right)
$$

and

$$
\int_{\Omega} \beta_{\epsilon}\left(u_{\epsilon}\right) T_{k}\left(u_{\epsilon}\right) d x \leq k\left(C(\mu, \Omega)+\|g\|_{L^{1}(\partial \Omega)}\right) .
$$

Let us prove (3.10). We use the fact that all integrals in (3.11) are nonnegative to obtain

$$
\lambda \int_{\partial \Omega} u_{\epsilon} T_{k}\left(u_{\epsilon}\right) d \sigma \leq k\left(C(\mu, g, \Omega)+\|g\|_{L^{1}(\partial \Omega)}\right) .
$$

We deduce from (3.12) that

$$
\int_{\partial \Omega} u_{\epsilon} \frac{1}{k} T_{k}\left(u_{\epsilon}\right) d \sigma \leq \frac{C(\mu, g, \Omega)+\|g\|_{L^{1}(\partial \Omega)}}{\lambda} .
$$

Finally, we pass to the limit as $k$ goes to 0 in (3.13) by using Fatou's lemma to get (3.10).

We have the following lemma (we refer to [27, Proposition 4.2]). 
Lemma 3.6. (i) The sequence $\left(\beta_{\epsilon}\left(u_{\epsilon}\right)\right)_{\epsilon>0}$ is uniformly bounded in $L^{1}(\Omega)$.

(ii) For any $k>0$, the sequence $\left(\beta_{\epsilon}\left(T_{k}\left(u_{\epsilon}\right)\right)_{\epsilon>0}\right.$ is uniformly bounded in $L^{1}(\Omega)$.

Proof.

(i) Dividing the terms in (3.9) by $k>0$ and letting $k$ goes to 0 , we get

$$
\lim _{k \rightarrow 0} \int_{\Omega} \beta_{\epsilon}\left(u_{\epsilon}\right) \frac{1}{k} T_{k}\left(u_{\epsilon}\right) d x=\int_{\Omega} \beta_{\epsilon}\left(u_{\epsilon}\right) \operatorname{sign}_{0}\left(u_{\epsilon}\right) d x .
$$

As

$$
\int_{\Omega} \beta_{\epsilon}\left(u_{\epsilon}\right) \operatorname{sign}_{0}\left(u_{\epsilon}\right) d x=\int_{\Omega}\left|\beta_{\epsilon}\left(u_{\epsilon}\right)\right| d x \leq C(\mu, \Omega)+\|g\|_{L^{1}(\partial \Omega)},
$$

then, (i) follows.

(ii) Assertion (ii) follows from (i). Since for any $k>0$,

$$
\int_{\Omega}\left|\beta_{\epsilon}\left(T_{k}\left(u_{\epsilon}\right)\right)\right| d x \leq \int_{\Omega}\left|\beta_{\epsilon}\left(u_{\epsilon}\right)\right| d x
$$

Proposition 3.7. Let $u_{\epsilon}$ be a weak solution of (3.4). Then, for all $k>0$,

$$
\sum_{i=1}^{N} \int_{\left|u_{\epsilon}\right| \leq k}\left|\frac{\partial u_{\epsilon}}{\partial x_{i}}\right|^{p_{i}^{-}} d x \leq N \operatorname{meas}(\Omega)+k\left(\frac{C(\mu, \Omega)+\|g\|_{L^{1}(\partial \Omega)}}{C_{3}}\right)
$$

and

$$
\int_{\partial \Omega}\left|T_{k}\left(u_{\epsilon}\right)\right| d \sigma \leq \frac{C(\mu, \Omega)+\|g\|_{L^{1}(\partial \Omega)}}{\lambda} .
$$

Proof. Let us prove (3.15).

$$
\begin{aligned}
\sum_{i=1}^{N} \int_{\left\{\left|u_{\epsilon}\right| \leq k\right\}}\left|\frac{\partial u_{\epsilon}}{\partial x_{i}}\right|^{p_{i}^{-}} d x= & \sum_{i=1}^{N} \int_{\left\{\left|u_{\epsilon}\right| \leq k,\left|\frac{\partial u_{\epsilon}}{\partial x_{i}}\right| \leq 1\right\}}\left|\frac{\partial u_{\epsilon}}{\partial x_{i}}\right|^{p_{i}^{-}} d x \\
& +\sum_{i=1}^{N} \int_{\left\{\left|u_{\epsilon}\right| \leq k,\left|\frac{\partial u_{\epsilon}}{\partial x_{i}}\right|>1\right\}}\left|\frac{\partial u_{\epsilon}}{\partial x_{i}}\right|^{p_{i}^{-}} d x \\
\leq & N \operatorname{meas}(\Omega)+\sum_{i=1}^{N} \int_{\left\{\left|u_{\epsilon}\right| \leq k,\left|\frac{\partial u_{\epsilon}}{\partial x_{i}}\right| \geq 1\right\}}\left|\frac{\partial u_{\epsilon}}{\partial x_{i}}\right|^{p_{i}(x)} d x \\
\leq & N \operatorname{meas}(\Omega)+\sum_{i=1}^{N} \int_{\left\{\left|u_{\epsilon}\right| \leq k\right\}}\left|\frac{\partial u_{\epsilon}}{\partial x_{i}}\right|^{p_{i}(x)} d x \\
\leq & N \operatorname{meas}(\Omega)+\sum_{i=1}^{N} \int_{\Omega}\left|\frac{\partial u_{\epsilon}}{\partial x_{i}}\right|^{p_{i}(x)} d x
\end{aligned}
$$




$$
\leq N \text { meas }(\Omega)+k\left(\frac{C(\mu, \Omega)+\|g\|_{L^{1}(\partial \Omega)}}{C_{3}}\right) .
$$

Since $\left|T_{k}\left(u_{\epsilon}\right)\right| \leq\left|u_{\epsilon}\right|$, we have

$$
\int_{\partial \Omega}\left|T_{k}\left(u_{\epsilon}\right)\right| d \sigma \leq\left\|u_{\epsilon}\right\|_{L^{1}(\partial \Omega)} .
$$

Then, we deduce from (3.10) and (3.17) that

$$
\int_{\partial \Omega}\left|T_{k}\left(u_{\epsilon}\right)\right| d \sigma \leq \frac{C(\mu, \Omega)+\|g\|_{L^{1}(\partial \Omega)}}{\lambda}
$$

Lemma 3.8. If $u_{\epsilon}$ is a weak solution of (3.4), then there exists a constant $D$ which depends on $\mu$ and $\Omega$ such that

$$
\operatorname{meas}\left\{\left|u_{\epsilon}\right|>k\right\} \leq \frac{D}{\min \left(b_{\epsilon}(k),\left|b_{\epsilon}(-k)\right|\right)}, \quad \forall k>0
$$

and a constant $D^{\prime}$ which depends on $\mu$ and $\Omega$ such that

$$
\text { meas }\left\{\left|\frac{\partial u_{\epsilon}}{\partial x_{i}}\right|>k\right\} \leq \frac{D^{\prime}}{k^{\frac{1}{\left(p_{M}^{-}\right)^{\prime}}}}, \forall k \geq 1 .
$$

Proof. For the proof of (3.18), we refer to [20, 27]. For the proof of (3.19), we refer to [6].

We need the following lemma (see $[6,17,18,22]$ ).

Lemma 3.9. For any $k>0$, there exists some positive constants $C$ and $C^{\prime}$ such that
(i) $\left\|u_{\epsilon}\right\|_{\mathcal{M}^{q^{*}(\Omega)}} \leq C$;
(ii) $\left\|\frac{\partial u_{\epsilon}}{\partial x_{i}}\right\|_{\mathcal{M}^{p^{-} \frac{q}{p}}(\Omega)} \leq C^{\prime}, \quad \forall i=1, \ldots, N$.

STEP 3 (Convergence results). In order to pass to the limit, the following convergence results are necessary (see [6] and [22]).

Lemma 3.10. For $i=1, \ldots, N$, as $\epsilon \rightarrow+\infty$, we have

$$
a_{i}\left(x, \frac{\partial u_{\epsilon}}{\partial x_{i}}\right) \longrightarrow a_{i}\left(x, \frac{\partial u}{\partial x_{i}}\right) \text { in } L^{1}(\Omega) \text { a.e. } x \in \Omega .
$$

Proposition 3.11. There exists a measurable function $u: \Omega \longrightarrow \mathbb{R}$ such that $u \in \operatorname{dom}(\beta)$ a.e. in $\Omega$ and

$$
u_{\epsilon} \longrightarrow u \text { in measure and a.e. in } \Omega \text { as } \epsilon \longrightarrow 0 .
$$


Proof. For the proof of (3.21), we refer to [6] (see also [22]).

As for $k>0, T_{k}$ is continuous, then $T_{k}\left(u_{\epsilon}\right) \rightarrow T_{k}(u)$ a.e. in $\Omega$. Finally, using Lemma 1.1 we deduce that for all $k>0, T_{k}(u) \in \operatorname{dom}(\beta)$ a.e. in $\Omega$. Since $T_{k}(u) \in \operatorname{dom}(\beta)$, we get $u \in \operatorname{dom}(\beta)$ a.e. in $\Omega$ and as $\operatorname{dom}(\beta)$ is bounded, then $u \in W^{1, \vec{p}(\cdot)}(\Omega)$.

Proposition 3.12. Assume (1.2)-(1.7). If $u_{\epsilon} \in E$ is a weak solution of (3.4) then

(i) for all $i=1, \ldots, N, \frac{\partial u_{\epsilon}}{\partial x_{i}}$ converges in measure to the weak partial gradient of $u$;

(ii) for all $i=1, \ldots, N$ and $k>0, a_{i}\left(x, \frac{\partial}{\partial x_{i}} T_{k}\left(u_{\epsilon}\right)\right)$ converges to $a_{i}\left(x, \frac{\partial}{\partial x_{i}} T_{k}(u)\right)$ in $L^{1}(\Omega)$ strongly and in $L^{p_{i}^{\prime}(\cdot)}(\Omega)$ weakly;

(iii) for $i=1, \ldots, N, a_{i}\left(x, \frac{\partial u_{n}}{\partial x_{i}}\right) \frac{\partial u_{\epsilon}}{\partial x_{i}} \longrightarrow a_{i}\left(x, \frac{\partial u}{\partial x_{i}}\right) \frac{\partial u}{\partial x_{i}}$ in $L^{1}(\Omega)$ and a.e. $x \in \Omega$.

Proof. For the proof of (i) and (ii) we refer to [6].

(iii) The continuity of $a_{i}(x, \xi)$ with respect to $\xi \in \mathbb{R}$ gives us

$$
a_{i}\left(x, \frac{\partial u_{n}}{\partial x_{i}}\right) \longrightarrow a_{i}\left(x, \frac{\partial u}{\partial x_{i}}\right) \text { a.e. } x \in \Omega \text {. }
$$

Therefore,

$$
a_{i}\left(x, \frac{\partial u_{\epsilon}}{\partial x_{i}}\right) \frac{\partial u_{\epsilon}}{\partial x_{i}} \longrightarrow a_{i}\left(x, \frac{\partial u}{\partial x_{i}}\right) \frac{\partial u}{\partial x_{i}} \text { a.e. } x \in \Omega .
$$

Setting $y_{\epsilon}=a_{i}\left(x, \frac{\partial u_{\epsilon}}{\partial x_{i}}\right) \frac{\partial u_{\epsilon}}{\partial x_{i}}$ and $y=a_{i}\left(x, \frac{\partial u}{\partial x_{i}}\right) \frac{\partial u}{\partial x_{i}}$, for $i=1, \ldots, N$, we have

$$
\left\{\begin{array}{l}
y_{\epsilon} \geq 0, y_{\epsilon} \rightarrow y \text { a.e. in } \Omega, y \in L^{1}(\Omega), \\
\int_{\Omega} y_{\epsilon} d x \rightarrow \int_{\Omega} y d x
\end{array}\right.
$$

and as

$$
\int_{\Omega}\left|y_{\epsilon}-y\right| d x=2 \int_{\Omega}\left(y-y_{\epsilon}\right)^{+} d x+\int_{\Omega}\left(y_{\epsilon}-y\right) d x
$$

and $\left(y-y_{\epsilon}\right)^{+} \leq y$, it follows by using Lebesgue dominated convergence theorem, that

which means that

$$
\lim _{\epsilon \rightarrow 0} \int_{\Omega}\left|y_{\epsilon}-y\right| d x=0
$$

$$
a_{i}\left(x, \frac{\partial u_{n}}{\partial x_{i}}\right) \frac{\partial u_{\epsilon}}{\partial x_{i}} \longrightarrow a_{i}\left(x, \frac{\partial u}{\partial x_{i}}\right) \frac{\partial u}{\partial x_{i}} \text { in } L^{1}(\Omega) \text { strongly. }
$$


We have the following lemmas (see [21, 25, 27]).

LEMma 3.13. For any $h \in C_{c}^{1}(\mathbb{R})$ and $\varphi \in W^{1, \vec{p}(\cdot)}(\Omega) \cap L^{\infty}(\Omega)$, for any $i=1, \ldots, N$,

$$
\frac{\partial}{\partial x_{i}}\left(h\left(u_{\epsilon}\right) \varphi\right) \longrightarrow \frac{\partial}{\partial x_{i}}(h(u) \varphi) \text { strongly in } L^{1}(\Omega) \text { as } \epsilon \rightarrow 0 .
$$

LEMMA 3.14. We have

$$
\begin{gathered}
\lim _{\epsilon \rightarrow 0} \int_{\Omega} h\left(u_{\epsilon}\right) \xi d \mu_{\epsilon}=\int_{\Omega} h(u) \xi d \mu, \\
\lim _{\epsilon \rightarrow 0} \epsilon \int_{\Omega}\left|u_{\epsilon}\right|^{p_{M}(x)-2} u_{\epsilon} h\left(u_{\epsilon}\right) \xi d x=0
\end{gathered}
$$

and

$$
\lim _{\epsilon \rightarrow 0} \lambda \int_{\partial \Omega} u_{\epsilon} h\left(u_{\epsilon}\right) \varphi d \sigma=\lambda \int_{\partial \Omega} u h(u) \varphi d \sigma,
$$

for any $h \in C_{c}^{1}(\mathbb{R})$ and $\varphi \in W^{1, \vec{p}(\cdot)}(\Omega) \cap L^{\infty}(\Omega)$.

Now, we pass to the limit in $\beta_{\epsilon}\left(u_{\epsilon}\right)$.

Since, for any $k>0,\left(h_{k}\left(u_{\epsilon}\right) \beta_{\epsilon}\left(u_{\epsilon}\right)\right)_{\epsilon>0}$ is bounded in $L^{1}(\Omega)$, there exists $z_{k} \in \mathcal{M}_{b}(\Omega)$ such that

$$
h_{k}\left(u_{\epsilon}\right) \beta_{\epsilon}\left(u_{\epsilon}\right) \rightarrow z_{k} \text { in } \mathcal{M}_{b}(\Omega) \text { as } \epsilon \rightarrow 0 .
$$

Moreover, for any $\varphi \in W^{1, \vec{p}(\cdot)}(\Omega) \cap L^{\infty}(\Omega)$, we have

$$
\begin{aligned}
\int_{\Omega} \varphi d z_{k}= & -\int_{\Omega} \sum_{i=1}^{N} a_{i}\left(x, \frac{\partial u}{\partial x_{i}}\right) \frac{\partial}{\partial x_{i}}\left[h_{k}(u) \varphi\right] d x \\
& -\lim _{\epsilon \rightarrow 0} \epsilon \int_{\Omega} \varphi|u|^{P_{M}(x)-2} u h_{k}(u) d x \\
& -\lambda \int_{\partial \Omega} \varphi u h_{k}(u) d \sigma+\int_{\Omega} \varphi h_{k}(u) d \mu+\int_{\partial \Omega} \varphi g h_{k}(u) d \sigma .
\end{aligned}
$$

Since

$$
-\lim _{\epsilon \rightarrow 0} \epsilon \int_{\Omega} \varphi|u|^{P_{M}(x)-2} u h_{k}(u) d x=0
$$

we have

$$
\begin{aligned}
\int_{\Omega} \varphi d z_{k}= & -\int_{\Omega} \sum_{i=1}^{N} a_{i}\left(x, \frac{\partial u}{\partial x_{i}}\right) \frac{\partial}{\partial x_{i}}\left[h_{k}(u) \varphi\right] d x-\lambda \int_{\partial \Omega} \varphi u h_{k}(u) d \sigma \\
& +\int_{\Omega} \varphi h_{k}(u) d \mu+\int_{\partial \Omega} \varphi g h_{k}(u) d \sigma
\end{aligned}
$$

which implies that $z_{k} \in \mathcal{M}_{b}^{p_{m}(\cdot)}(\Omega)$ and, for any $k \leq l$,

$$
z_{k}=z_{l} \text { on }\left[\left|T_{k}(u)\right|<k\right] \text {. }
$$


Let us consider the Radon measure defined by

$$
\left\{\begin{array}{l}
z=z_{k}, \quad \text { on }\left[\left|T_{k}(u)\right|<k\right] \text { for } k \in \mathbb{N}^{*}, \\
z=0 \text { on } \bigcap_{k \in \mathbb{N}^{*}}\left[\left|T_{k}(u)\right|=k\right] .
\end{array}\right.
$$

For any $h \in \mathcal{C}_{c}(\mathbb{R}), h(u) \in L^{\infty}(\Omega, d|z|)$ and

$$
\begin{aligned}
\int_{\Omega} h(u) \varphi d z= & -\int_{\Omega} \sum_{i=1}^{N} a_{i}\left(x, \frac{\partial u}{\partial x_{i}}\right) \frac{\partial}{\partial x_{i}}[h(u) \varphi] d x-\lambda \int_{\partial \Omega} u h(u) \varphi d \sigma \\
& +\int_{\Omega} h(u) \varphi d \mu+\int_{\partial \Omega} g h(u) \varphi d \sigma
\end{aligned}
$$

for any $\varphi \in W^{1, \vec{p}(\cdot)}(\Omega) \cap L^{\infty}(\Omega)$.

Indeed, let $k_{0}>0$ be such that $\operatorname{supp}(h) \subseteq\left[-k_{0}, k_{0}\right]$,

$$
\begin{aligned}
\int_{\Omega} h(u) \xi d z=\int_{\Omega} h(u) \xi d z_{k_{0}} \\
=-\lim _{\epsilon \rightarrow 0} \int_{\Omega} \sum_{i=1}^{N} a_{i}\left(x, \frac{\partial u_{\epsilon}}{\partial x_{i}}\right) \frac{\partial}{\partial x_{i}}\left[h\left(u_{\epsilon}\right) \varphi\right] d x \\
\quad-\lim _{\epsilon \rightarrow 0} \epsilon \int_{\Omega}\left|u_{\epsilon}\right|^{p_{M}(x)-2} u_{\epsilon} h\left(u_{\epsilon}\right) \varphi d x-\lim _{\epsilon \rightarrow 0} \lambda \int_{\partial \Omega} u_{\epsilon} h\left(u_{\epsilon}\right) \varphi d \sigma \\
\quad+\lim _{\epsilon \rightarrow 0} \int_{\Omega} h\left(u_{\epsilon}\right) \varphi d \mu_{\epsilon}+\lim _{\epsilon \rightarrow 0} \int_{\partial \Omega} g_{\epsilon} h\left(u_{\epsilon}\right) \varphi d \sigma \\
=-\lim _{\epsilon \rightarrow 0} \int_{\Omega} \sum_{i=1}^{N} a_{i}\left(x, \frac{\partial T_{k}\left(u_{\epsilon}\right)}{\partial x_{i}}\right) \frac{\partial}{\partial x_{i}}\left[h\left(u_{\epsilon}\right) \varphi\right] d x \\
\quad-\lim _{\epsilon \rightarrow 0} \epsilon \int_{\Omega}\left|u_{\epsilon}\right|^{p_{M}(x)-2} u_{\epsilon} h\left(u_{\epsilon}\right) \varphi d x-\lim _{\epsilon \rightarrow 0} \lambda \int_{\partial \Omega} u_{\epsilon} h\left(u_{\epsilon}\right) \varphi d \sigma \\
\quad+\lim _{\epsilon \rightarrow 0} \int_{\Omega} h\left(u_{\epsilon}\right) \varphi d \mu_{\epsilon}+\lim _{\epsilon \rightarrow 0} \int_{\partial \Omega} g_{\epsilon} h\left(u_{\epsilon}\right) \varphi d \sigma \\
=-\int_{\Omega} \sum_{i=1}^{N} a_{i}\left(x, \frac{\partial u}{\partial x_{i}}\right) \frac{\partial}{\partial x_{i}}[h(u) \xi] d x-\lambda \int_{\partial \Omega} u h(u) \varphi d \sigma \\
\quad+\int_{\Omega} h(u) \varphi d \mu+\int_{\partial \Omega} g h(u) \varphi d \sigma .
\end{aligned}
$$

Moereover, we have the following lemma (see [25, Lemma 4.7]).

Lemma 3.15. The Radon-Nikodym decomposition of the measure z given by (3.25) with respect to $\mathcal{L}^{N}$,

$$
z=b \mathcal{L}^{N}+\nu \text { with } \nu \perp \mathcal{L}^{N}
$$


satisfies the following properties

$$
\left\{\begin{array}{l}
b \in \beta(u) \mathcal{L}^{N}-\text { a.e. in } \Omega, b \in L^{1}(\Omega), \nu \in \mathcal{M}_{b}^{p_{i}(\cdot)}(\Omega), \\
\nu^{+} \text {is concentrated on }[u=M], \\
\nu^{-} \text {is concentrated on }[u=m] .
\end{array}\right.
$$

To end the proof of Theorem 3.2, we consider $\varphi \in W^{1, \vec{p}(\cdot)}(\Omega) \cap L^{\infty}(\Omega)$ and $h \in C_{c}^{1}(\mathbb{R})$. Then, we take $h\left(u_{\epsilon}\right) \varphi$ as a test function in (3.5) to get

$$
\begin{aligned}
& \sum_{i=1}^{N} \int_{\Omega} a_{i}\left(x, \frac{\partial u_{\epsilon}}{\partial x_{i}}\right) \frac{\partial}{\partial x_{i}}\left[h\left(u_{\epsilon}\right) \varphi\right] d x+\int_{\Omega} \beta_{\epsilon}\left(u_{\epsilon}\right) h\left(u_{\epsilon}\right) \varphi d x \\
& \quad+\epsilon \int_{\Omega}\left|u_{\epsilon}\right|^{p_{M}(x)-2} u_{\epsilon} h\left(u_{\epsilon}\right) \varphi d x+\lambda \int_{\partial \Omega} u_{\epsilon} h\left(u_{\epsilon}\right) \varphi d \sigma \\
& =\int_{\Omega} h\left(u_{\epsilon}\right) \varphi d \mu_{\epsilon}+\int_{\partial \Omega} g_{\epsilon} h\left(u_{\epsilon}\right) \varphi d \sigma .
\end{aligned}
$$

The first term of (3.26) can be written as

$$
\begin{array}{r}
\sum_{i=1}^{N} \int_{\Omega} a_{i}\left(x, \frac{\partial T_{l_{0}+1}\left(u_{\epsilon}\right)}{\partial x_{i}}\right) \frac{\partial}{\partial x_{i}}\left[h\left(u_{\epsilon}\right) \varphi\right] d x \\
=\sum_{i=1}^{N} \int_{\Omega} a_{i}\left(x, \frac{\partial u_{\epsilon}}{\partial x_{i}}\right) \frac{\partial}{\partial x_{i}}\left[h\left(u_{\epsilon}\right) \varphi\right] d x
\end{array}
$$

for some $l_{0}>0$; so, by Lemmas 3.13 and 3.14, we have

$$
\begin{aligned}
\lim _{\epsilon \rightarrow 0} & \int_{\Omega} \sum_{i=1}^{N} a_{i}\left(x, \frac{\partial u_{\epsilon}}{\partial x_{i}}\right) \frac{\partial}{\partial x_{i}}\left[h\left(u_{\epsilon}\right) \xi\right] d x \\
& =\lim _{\epsilon \rightarrow 0} \int_{\Omega} \sum_{i=1}^{N} a_{i}\left(x, \frac{\partial T_{l_{0}+1}\left(u_{\epsilon}\right)}{\partial x_{i}}\right) \frac{\partial}{\partial x_{i}}\left[h\left(u_{\epsilon}\right) \xi\right] d x \\
& =\int_{\Omega} \sum_{i=1}^{N} a_{i}\left(x, \frac{\partial T_{l_{0}+1}(u)}{\partial x_{i}}\right) \frac{\partial}{\partial x_{i}}[h(u) \xi] d x \\
& =\int_{\Omega} \sum_{i=1}^{N} a_{i}\left(x, \frac{\partial u}{\partial x_{i}}\right) \frac{\partial}{\partial x_{i}}[h(u) \xi] d x .
\end{aligned}
$$

By using convergence results in (3.26), we get

$$
\begin{aligned}
\lim _{\epsilon \rightarrow 0} \int_{\Omega} \beta_{\epsilon}\left(u_{\epsilon}\right) h\left(u_{\epsilon}\right) \xi d x= & \int_{\Omega} h(u) \xi d \mu+\int_{\partial \Omega} g h(u) \varphi d \sigma-\lambda \int_{\partial \Omega} u h(u) \varphi d \sigma \\
& -\sum_{i=1}^{N} \int_{\Omega} a_{i}\left(x, \frac{\partial u}{\partial x_{i}}\right) \frac{\partial}{\partial x_{i}}[h(u) \xi] d x
\end{aligned}
$$




$$
=\int_{\Omega} h(u) \varphi d z=\int_{\Omega} b h(u) \varphi d x+\int_{\Omega} h(u) \varphi d \nu .
$$

Passing to the limit in (3.26) as $\epsilon \rightarrow 0$, we get

$$
\begin{gathered}
\sum_{i=1}^{N} \int_{\Omega} a_{i}\left(x, \frac{\partial u}{\partial x_{i}}\right) \frac{\partial}{\partial x_{i}}[h(u) \varphi] d x+\int_{\Omega} b h(u) \varphi d x+\int_{\Omega} h(u) \varphi d \nu \\
+\lambda \int_{\partial \Omega} u h(u) \varphi d \sigma=\int_{\Omega} h(u) \varphi d \mu+\int_{\partial \Omega} g h(u) \varphi d \sigma .
\end{gathered}
$$

Letting $\epsilon$ goes to 0 in $(3.26)$ it yields that $(b, u)$ is a solution of the problem (1.1). To end the proof of Theorem 3.2, we prove (3.2). We take $\xi=T_{1}\left(u_{\epsilon}-\right.$ $\left.T_{n}\left(u_{\epsilon}\right)\right)$ as a test function in (3.5) to get

$$
\begin{aligned}
& \sum_{i=1}^{N} \int_{\Omega} a_{i}\left(x, \frac{\partial u_{\epsilon}}{\partial x_{i}}\right) \frac{\partial}{\partial x_{i}}\left(T_{1}\left(u_{\epsilon}-T_{n}\left(u_{\epsilon}\right)\right)\right) d x+\int_{\Omega} \beta_{\epsilon}\left(u_{\epsilon}\right) T_{1}\left(u_{\epsilon}-T_{n}\left(u_{\epsilon}\right)\right) d x \\
& \quad+\epsilon \int_{\Omega}\left|u_{\epsilon}\right|^{P_{M}(x)-2} u_{\epsilon} T_{1}\left(u_{\epsilon}-T_{n}\left(u_{\epsilon}\right)\right) d x+\lambda \int_{\partial \Omega} u_{\epsilon} T_{1}\left(u_{\epsilon}-T_{n}\left(u_{\epsilon}\right)\right) d \sigma \\
& \quad=\int_{\Omega} T_{1}\left(u_{\epsilon}-T_{n}\left(u_{\epsilon}\right)\right) d \mu_{\epsilon}+\int_{\Omega} g_{\epsilon} T_{1}\left(u_{\epsilon}-T_{n}\left(u_{\epsilon}\right)\right) d x
\end{aligned}
$$

Since

$$
\begin{gathered}
\int_{\Omega} \beta_{\epsilon}\left(u_{\epsilon}\right) T_{1}\left(u_{\epsilon}-\right. \\
\left.T_{n}\left(u_{\epsilon}\right)\right) d x+\epsilon \int_{\Omega}\left|u_{\epsilon}\right|^{P_{M}(x)-2} u_{\epsilon} T_{1}\left(u_{\epsilon}-T_{n}\left(u_{\epsilon}\right)\right) d x \\
+\lambda \int_{\partial \Omega} u_{\epsilon} T_{1}\left(u_{\epsilon}-T_{n}\left(u_{\epsilon}\right)\right) d \sigma \geq 0
\end{gathered}
$$

and

$$
\frac{\partial}{\partial x_{i}}\left(T_{1}\left(u_{\epsilon}-T_{n}\left(u_{\epsilon}\right)\right)\right)=\frac{\partial u_{\epsilon}}{\partial x_{i}} \chi_{\left[n<\left|u_{\epsilon}\right|<n+1\right]}
$$

we have from equality (3.28),

$$
\begin{aligned}
& \sum_{i=1}^{N} \int_{\left[n<\left|u_{\epsilon}\right|<n+1\right]} a_{i}\left(x, \frac{\partial u_{\epsilon}}{\partial x_{i}}\right) \frac{\partial u_{\epsilon}}{\partial x_{i}} d x \\
& \quad \leq \int_{\Omega} T_{1}\left(u_{\epsilon}-T_{n}\left(u_{\epsilon}\right)\right) d \mu_{\epsilon}+\int_{\Omega} g_{\epsilon} T_{1}\left(u_{\epsilon}-T_{n}\left(u_{\epsilon}\right)\right) d x
\end{aligned}
$$

We have (see [27])

$$
\lim _{n \rightarrow+\infty} \lim _{\epsilon \rightarrow 0} \int_{\Omega} T_{1}\left(u_{\epsilon}-T_{n}\left(u_{\epsilon}\right)\right) d \mu_{\epsilon}=0
$$

and

$$
\lim _{n \rightarrow+\infty} \lim _{\epsilon \rightarrow 0} \int_{\Omega} g_{\epsilon} T_{1}\left(u_{\epsilon}-T_{n}\left(u_{\epsilon}\right)\right) d x=0
$$


Then, using (1.5), and letting $n \rightarrow+\infty, \epsilon \rightarrow 0$ respectively in (3.12), we get

$$
\begin{aligned}
\lim _{n \rightarrow+\infty} & \lim _{\epsilon \rightarrow 0} \frac{1}{C} \sum_{i=1}^{N} \int_{\left[n<\left|u_{\epsilon}\right|<n+1\right]}\left|\frac{\partial u_{\epsilon}}{\partial x_{i}}\right|^{p_{i}(x)} d x \\
= & \lim _{n \rightarrow+\infty} \frac{1}{C} \sum_{i=1}^{N} \int_{[n<|u|<n+1]}\left|\frac{\partial u}{\partial x_{i}}\right|^{p_{i}(x)} d x \leq 0 .
\end{aligned}
$$

The connection between our notion of solution and the entropic formulation is given in the following Theorem. In particular, as the domain of $\beta$ is bounded, this equivalent formulation is very useful for the proof of uniqueness of solution to problem (1.1). We reason as in [25] to get the following results.

THEOREM 3.16. If $(u, b)$ is a solution of (1.1) in the sense of Theorem 3.2, then $(u, b)$ is a solution in the following sense: for any $\varphi \in W^{1, \vec{p}(\cdot)}(\Omega) \cap$ $L^{\infty}(\Omega)$ such that $\varphi \in \operatorname{dom} \beta$ and for any $k>0$

$$
\begin{aligned}
& \sum_{i=1}^{N} \int_{\Omega} a_{i}\left(x, \frac{\partial u}{\partial x_{i}}\right) \frac{\partial}{\partial x_{i}} T_{k}(u-\varphi) d x+\int_{\Omega} b T_{k}(u-\varphi) d x \\
& \quad+\lambda \int_{\partial \Omega} u T_{k}(u-\varphi) d \sigma \\
& \quad \leq \int_{\Omega} T_{k}(u-\varphi) d \mu+\int_{\partial \Omega} g T_{k}(u-\varphi) d \sigma
\end{aligned}
$$

The result of the uniqueness of solution to problem (1.1) is the following.

Theorem 3.17. Let $\left(u_{1}, b_{1}\right)$ and $\left(u_{2}, b_{2}\right)$ be two solutions of (1.1). Then

$$
\begin{cases}u_{1}=u_{2} \text { a.e. } & \text { in } \Omega \\ b_{1}=b_{2} \text { a.e. } & \text { in } \Omega .\end{cases}
$$

Proof. For $u_{1}$, we choose $\varphi=u_{2}$ as a test function in (3.30) to get

$$
\begin{aligned}
& \sum_{i=1}^{N} \int_{\Omega} a_{i}\left(x, \frac{\partial u_{1}}{\partial x_{i}}\right) \frac{\partial}{\partial x_{i}} T_{k}\left(u_{1}-u_{2}\right) d x+\int_{\Omega} b_{1} T_{k}\left(u_{1}-u_{2}\right) d x \\
& \quad+\lambda \int_{\partial \Omega} u_{1} T_{k}\left(u_{1}-u_{2}\right) d \sigma \leq \int_{\partial \Omega} g T_{k}\left(u_{1}-u_{2}\right) d \sigma+\int_{\Omega} T_{k}\left(u_{1}-u_{2}\right) d \mu .
\end{aligned}
$$

Similarly for $u_{2}$, we choose $\varphi=u_{1}$ as a test function in (3.30) to get

$$
\begin{aligned}
& \sum_{i=1}^{N} \int_{\Omega} a_{i}\left(x, \frac{\partial u_{2}}{\partial x_{i}}\right) \frac{\partial}{\partial x_{i}} T_{k}\left(u_{2}-u_{1}\right) d x+\int_{\Omega} b_{2} T_{k}\left(u_{2}-u_{1}\right) d x \\
& \quad+\lambda \int_{\partial \Omega} u_{2} T_{k}\left(u_{2}-u_{1}\right) d \sigma \leq \int_{\Omega} T_{k}\left(u_{2}-u_{1}\right) d \mu+\int_{\partial \Omega} g T_{k}\left(u_{2}-u_{1}\right) d \sigma
\end{aligned}
$$


Adding these two inequalities yields

$$
\begin{aligned}
& \sum_{i=1}^{N} \int_{\Omega}\left(a_{i}\left(x, \frac{\partial u_{1}}{\partial x_{i}}\right)-a_{i}\left(x, \frac{\partial u_{2}}{\partial x_{i}}\right)\right) \frac{\partial}{\partial x_{i}} T_{k}\left(u_{1}-u_{2}\right) d x \\
& \quad+\int_{\Omega}\left(b_{1}-b_{2}\right) T_{k}\left(u_{1}-u_{2}\right) d x+\lambda \int_{\partial \Omega}\left(u_{1}-u_{2}\right) T_{k}\left(u_{1}-u_{2}\right) d \sigma \leq 0 .
\end{aligned}
$$

Therefore, as in [18] the result follows.

\section{REFERENCES}

[1] L. Alvarez, P.-L. Lions, and J.-M. Morel, Image selective smoothing and edge detection by nonlinear diffusion. II, SIAM J. Numer. Anal. 29 (1992), 845-866.

[2] M. Bendahmane, M. Langlais and M. Saad, On some anisotropic reaction- diffusion systems with $L^{1}$-data modeling the propagation of an epidemic disease, Nonlinear Anal. 54 (2003), 617-636.

[3] L. Boccardo and T. Gallouët, Nonlinear elliptic and parabolic equations involving measure data, J. Funct. Anal. 87 (1989), 149-169.

[4] L. Boccardo and T. Gallouët, Nonlinear elliptic equations with right hand side measures, Comm. Partial Differential Equations 17 (1992), 641-655.

[5] L. Boccardo, T. Gallouët and L. Orsina, Existence and uniqueness of entropy solution for nonlinear elliptic equations with measure data, Ann. Inst. Henri Poincaré Anal. Non Linéaire 13 (1996), 539-551.

[6] B. Koné, S. Ouaro and F.D.Y. Zongo, Nonlinear elliptic anisotropic problem with Fourier boundary condition, Int. J. Evol. Equ. 8 (2013), 305-328.

[7] M.-M. Boureanu and V. D. Radulescu, Anisotropic Neumann problems in Sobolev spaces with variable exponent, Nonlinear Anal. 75 (2012), 4471-4482.

[8] H. Brézis, Analyse fonctionnelle. Théorie et applications, Masson, Paris, 1983.

[9] H. Brézis, Opérateurs maximaux monotones et semi-groupes de contractions dans les espaces de Hilbert, North Holland, Amsterdam, 1973.

[10] Y. Chen, S. Levine and M. Rao, Variable exponent, linear growth functionals in image restoration, SIAM J. Appl. Math. 66 (2006), 1383-1406.

[11] G. Dal Maso, F. Murat, L. Orsina and A. Prignet, Renormalized solutions of elliptic equations with general measure data, Ann. Scuola Norm. Sup. Pisa Cl. Sci. (4) 28 (1999), 741-808.

[12] L. Diening, Theoretical and numerical results for electrorheological fluids, PhD. thesis, University of Frieburg, Germany, 2002.

[13] G. Dolzmann, N. Hungerbühler and S. Müller, Non-linear elliptic systems with measure-valued right-hand side, Math Z. 226 (1997), 545-574.

[14] F. Ettwein and M. Ruzicka, Existence of strong solutions for electrorheological fluids in two dimensions: steady Dirichlet problem, in: Geometric Analysis and Nonlinear Partial Differential Equations, Springer-Verlag, Berlin, 2003, 591-602.

[15] X. Fan, Anisotropic variable exponent Sobolev spaces and $\vec{p}(\cdot)$-Laplacian equations, Complex Var. Elliptic Equ. 56 (2011), 623-642.

[16] X. Fan and D. Zhao, On the spaces $L^{p(x)}(\Omega)$ and $W^{m, p(x)}(\Omega)$, J. Math. Anal. Appl. 263 (2001), 424-446.

[17] I. Ibrango and S. Ouaro, Entropy solutions for anisotropic nonlinear problems with homogeneous Neumann boundary condition, J. Appl. Anal. Comput. 6 (2016), 271292. 
[18] I. Ibrango and S. Ouaro, Entropy solution for doubly nonlinear elliptic anisotropic problems with Fourier boundary conditions, Discuss. Math. Differ. Incl. Control Optim. 35 (2015), 123-150.

[19] N. Igbida, S. Ouaro and S. Soma, Elliptic problem involving diffuse measure data, J. Differential Equations 253 (2012), 3159-3183.

[20] I. Konaté and S. Ouaro, Good Radon measure for anisotropic problems with variable exponent, Electron. J. Differential Equations 2016 (2016), No. 221, 19 pp.

[21] I. Konaté and S. Ouaro, Nonlinear multivalued problems with variable exponent and diffuse measure data in anisotropic space, Gulf J. Math. 6 (2018), 13-30.

[22] B. Koné, S. Ouaro and S. Soma, Weak solutions for anisotropic nonlinear elliptic problem with variable exponent and measure data, Int. J. Evol. Equ. 5 (2010), 327350 .

[23] O. Kovacik and J. Rakosnik, On spaces $L^{p(x)}$ and $W^{k, p(x)}$, Czechoslovak Math. J. 41(116) (1991), 592-618.

[24] M. Mihailescu, P. Pucci and V. Radulescu, Eigenvalue problems for anisotropic quasilinear elliptic equations with variable exponent, J. Math. Anal. Appl. 340 (2008), 687-698.

[25] I. Nyanquini, S. Ouaro and S. Soma, Entropy solution to nonlinear multivalued elliptic problem with variable exponents and measure data, An. Univ. Craiova Ser. Mat. Inform. 40 (2013), 174-198.

[26] I. Nyanquini, S. Ouaro, Entropy solution for nonlinear elliptic problem involving variable exponent and Fourier type boundary condition, Afr. Mat. 23 (2012), 205-228.

[27] S. Ouaro, A. Ouédraogo and S. Soma, Multivalued homogeneous Neumann problem involving diffuse measure data and variable exponent, Nonlinear Dyn. Syst. Theory 16 (2016), 102-114.

[28] C. Pfeiffer, C. Mavroidis, Y. Bar-Cohen, B. Dolgin, Electrorheological fluid based force feedback device, in Proc. SPIE 3840, Telemanipulator and Telepresence Technologies VI, 1999, 88-99.

[29] K. Rajagopal and M. Ružička, Mathematical modelling of electro-rheological fluids, Cont. Mech. Therm. 13 (2001), 59-78.

[30] M. Ružička, Electrorheological fluids: modelling and mathematical theory, Springer, Berlin, 2000.

[31] M. Troisi, Teoremi di inclusione per spazi di Sobolev non isotropi, Recherche. Mat 18 (1969), 3-24.

I. Konaté

Laboratoire de Mathématiques et Informatique, UFR. Sciences Exactes et Appliquées Université Joseph Ki Zerbo

03 BP 7021 Ouaga 03, Ouagadougou

Burkina Faso

E-mail: ibrakonat@yahoo.fr

S. Ouaro

Laboratoire de Mathématiques et Informatique, UFR. Sciences Exactes et Appliquées Université Joseph Ki Zerbo

03 BP 7021 Ouaga 03, Ouagadougou

Burkina Faso

E-mail: ouaro@yahoo.fr

Received: 12.3.2018.

Revised: 27.10 .2018 . 